\title{
How to Deal with the Issues of Fertility, Malignancies, and the Postfinasteride Syndrome while Prescribing Finasteride for Male Pattern Hair Loss
}

\author{
Ralph M. Trüeb ${ }^{a}$ Ngoc-Nhi Catharina Luu ${ }^{a}$ \\ Maria Fernanda Reis Gavazzoni Dias ${ }^{b}$ Hudson Dutra Rezende ${ }^{c}$ \\ ${ }^{a}$ Center for Dermatology and Hair Diseases Professor Trüeb, Wallisellen, Switzerland; ${ }^{b}$ Department of Dermatology, \\ Universidade Federal Fluminense, Centro de Ciências Médicas, Hospital Universitário Antonia Pedro, Rio de Janeiro, \\ Brazil; ' Department of Dermatology, Alvaro Alvim School Hospital, Campos dos Goytacazes, Rio de Janeiro, Brazil
}

\section{Keywords}

Finasteride · Fertility · Prostate cancer · Breast cancer · Postfinasteride syndrome $\cdot$ Sperm count $\cdot$ Prostate-specific antigen · Gynecomastia · Histrionic personality disorder diagnostic criteria

\begin{abstract}
Oral finasteride represented a breakthrough for treatment of male pattern hair loss (MPHL), with clinical studies having demonstrated high efficacy of treatment and a favorable safety profile. And yet, fertility issues, malignancy, and postfinasteride syndrome have been concerns of users and prescribers of the drug. Pre-existing mental health disorder may put patients at an increased risk of nocebo, while the prevalence of personality disorders in subjects with MPHL is known to be higher than in the general population, specifically histrionic personality disorder. We devised a system for patient selection and risk assessment, including fertility issues, regular PSA determinations, and specific mental health assessment. For those who choose regular prostate cancer screening, the use of finasteride meaningfully reduces the risk of prostate cancer. While gynecomastia is a known, rare
\end{abstract}

adverse effect of finasteride, so far, studies support the view that exposure to finasteride is not associated with male breast cancer risk. Patient understanding and involvement are central to optimal treatment selection and active patient role in treatment.

(c) 2022 S. Karger AG, Basel

Oral finasteride represented a major breakthrough in the treatment of male pattern hair loss (MPHL), based on an understanding of the underlying pathophysiology [1] and observations on the respective genetic defect of 5-alpha reductase [2]. Clinical studies have demonstrated both a high efficacy of treatment and a favorable safety profile [3-10], establishing the drug as the first-line treatment of MPHL. In 2012, Sato and Takeda [11] reported on efficacy and safety of $1 \mathrm{mg}$ oral finasteride for treatment of MPHL in the so far largest population study of enrolled 3,177 Japanese men. The overall effect on hair growth was seen in $87.1 \%$, in whom hair increased greatly in $11.1 \%$, moderately in $36.5 \%$, and slightly in $39.5 \%$. The response rate improved with increasing duration of treatment. Adverse reactions occurred in $0.7 \%$ of men. No specific safety problems associated with long-term use 
Table 1. Issues to be addressed while prescribing finasteride for treatment of male pattern hair loss

Patient selection and risk assessment

Appropriate patient information

How to react in case of drug-related adverse events

Issues of fertility and of malignancy (prostate and breast)

Management of the PFS

Alternative treatments

were observed. The authors concluded that in Japanese men with MPHL, $1 \mathrm{mg}$ oral finasteride used for long-term treatment maintains progressive hair regrowth without recognized side effect.

And yet, particularly fertility issues and postfinasteride syndrome (PFS) have become concerns of both users and prescribers of the drug, sensitized by social media coverage following the respective reports on decreased fertility $[12,13]$ and persistent sexual side effects after cessation of finasteride therapy [14-20]. While Overstreet et al. [21] have demonstrated that treatment with $1 \mathrm{mg}$ finasteride daily for 48 weeks did not affect spermatogenesis or semen production in young men, there have been case reports of a negative effect of oral finasteride on men with pre-existing decreased fertility $[12,13]$.

Meanwhile, PFS is claimed to occur in men who have taken oral finasteride with reported symptoms supposedly persisting despite quitting finasteride. These include loss of libido, erectile dysfunction, reduction in penis size, change in penile curvature, reduced sensation, gynecomastia, muscle atrophy, cognitive impairment, severely dry skin, and depression [14-20]. The condition allegedly may have a life-altering impact on sufferers, while also being linked to depression and suicidality [22].

The condition is not recognized by the scientific community. And yet, individuals who suffer from the syndrome do present with very distinctive and relatively homogenous symptoms that cannot be simply dismissed as nonexistent. Ultimately, it is not sufficient to only discuss the plausibility of PFS [23], not alone to ridicule its proponents [24]. Rather, there is a need for a pragmatic approach to PFS to include such important issues as listed in Table 1 (from [25]).

In fact, after a 20 -year history of successful prescription of finasteride for treatment of MPHL, we eventually reported our first case of PFS with circumstantial evidence that the condition may rather have represented a delusional disorder of the somatic type than an endocrinological disorder, possibly on the background of a histrionic personality disorder [26]. Besides, the PFS dem- onstrates some striking analogies to other mystery syndromes, such as amalgam illness, multiple chemical sensitivity, Morgellons disease, and Koro, in which the alleged symptoms cannot be explained biologically and the frequency of consultations for the respective condition strikingly parallels the media coverage, which points to a high degree of suggestibility.

Consequently, we devised a system for patient selection and risk assessment, including fertility issues, regular prostate-specific antigen determinations, and specific mental health assessment. Pre-existing mental health disorder may put patients at an increased risk of nocebo, while the prevalence of personality disorders in subjects with MPHL is known to be significantly higher than in the general population [27], specifically the histrionic personality disorder.

The diagnosis of a histrionic personality disorder is based on specific criteria in the Diagnostic and Statistical Manual of Mental Disorders, Fifth Edition (DSM-5), published by the American Psychiatric Association. To diagnose histrionic personality disorder, people must persistently exaggerate their emotions and seek attention, as shown by at least 5 of the symptoms listed in Table 2.

These questions can be addressed with a respective checklist together with the patient at hand. At least in those men who are hesitant regarding their fertility, if not generally in all men, we recommend assessing fertility with an andrologist. Alternatively, for those feeling uncomfortable doing so, the sperm count can be evaluated in privacy with one of the respective commercially available kits. In doubtful cases, the latter does not replace a comprehensive andrologic examination.

Finally, in men 45 and over, regular prostate-specific antigen screening before and after starting therapy with oral finasteride, and thereafter twice yearly, has been recommended. The level should drop by ca. $50 \%$ upon initiation of therapy. In case of increase $>0.4 \mathrm{ng} / \mathrm{mL}$ per year, the prostate should be further evaluated. Ultimately, for those who choose regular prostate cancer screening, the use of finasteride meaningfully reduces the risk of prostate cancer and thus the morbidity associated with treatment of the disease [28].

While gynecomastia (both unilateral and bilateral) is a known though relatively rare adverse effect of oral finasteride for treatment of MPHL [29], so far, the respective studies also support the view that exposure to finasteride is not associated materially with male breast cancer risk $[30,31]$. Of course, in case of sexual adverse effects, testicular tenderness, mood disorder, gynecomastia, and de- 
Table 2. Diagnostic criteria for histrionic personality disorder
They feel uncomfortable when they are not the center of attention

They interact with others in inappropriately sexually seductive or provocative ways

Their emotions shift rapidly, making them seem shallow

They consistently use their physical appearance to call attention to themselves

Their speech is extremely vague, lacking detail

They express their emotions in a dramatic, theatrical, and extravagant way

They are easily influenced by others or situations

They view relationships as more intimate than the relationships are creased fertility, treatment with oral finasteride should be suspended.

To finish, with regard to management of PFS, attention must be focused on the treatment of depression and sexual symptoms. Since there is no evidence of androgen deficiency, persistent steroid 5-alpha reductase inhibition, or androgen insensitivity, symptomatic finasteride users are unlikely to benefit from treatment with testosterone, dihydrotestosterone, or any other androgen [32].

Patient understanding and involvement are central to optimal treatment selection and active patient role in treatment. Patients frequently become preoccupied with potential side effects when they are reluctant to undergo treatment, and some physicians also overestimate the side effects.

A significant nocebo effect has been demonstrated in patients who were informed of potential sexual adverse effects before taking finasteride versus patients who were not informed [33]. Nevertheless, it is of utmost importance to inform patients on potential adverse effects, their frequencies, and appropriate management.

Yet, the real concern should be the underlying medical condition that is often displaced in the patient's mind by fear of the treatment. Patients must adopt a broader perspective, the long view, and not a vision narrowed by fear. The physician's role is to help the patient figure out what he really wants and then to use the power of persuasion to show the patient the way there. At length, the way a physician phrases his recommendations can powerfully sway a patient's choice and have influence on the treatment outcome, both in terms of efficacy and of tolerability [34].

\section{Statement of Ethics}

The authors have no ethical conflicts to disclose.

\section{Conflict of Interest Statement}

The authors declare that they have no conflicts of interest to disclose.

\section{Funding Sources}

The authors have received no external funding.

\section{Author Contributions}

Professors Trüeb and Gavazzoni take responsibility for the integrity of the data and the accuracy of the data analysis. Trüeb, Luu, Gavazzoni, and Rezende contributed to study concept and design. Trüeb, Luu Gavazzoni, and Dutra contributed to critical revision of the manuscript for important intellectual content.

\section{References}

1 Kaufman KD. Androgen metabolism as it affects hair growth in androgenetic alopecia. Dermatol Clin. 1996;14:697-711.

2 Imperato-McGinley J, Guerrero L, Gautier T, Peterson RE. Steroid 5-alpha-reductase deficiency in man: an inherited form of male pseudohermaphroditism. Science. 1974;186: 1213-5.

3 Kaufman KD, Olsen EA, Whiting D, Savin R, DeVillez R, Bergfeld W, et al. Finasteride in the treatment of men with androgenetic alopecia. Finasteride Male Pattern Hair Loss Study Group. J Am Acad Dermatol. 1998;39:578-89.
4 Leyden J, Dunlap F, Miller B, Winters P, Lebwohl M, Hecker D, et al. Finasteride in the treatment of men with frontal male pattern hair loss. J Am Acad Dermatol. 1999;40:9307.

5 Whiting DA, Waldstreicher J, Sanchez M, Kaufman KD. Measuring reversal of hair miniaturization in androgenetic alopecia by follicular counts in horizontal sections of serial scalp biopsies: results of finasteride $1 \mathrm{mg}$ treatment of men and postmenopausal women. J Investig Dermatol Symp Proc. 1999;4: 282-4.
6 Van Neste D, Fuh V, Sanchez-Pedreno P, Lopez-Bran E, Wolff H, Whiting D, et al. Finasteride increases anagen hair in men with androgenetic alopecia. Br J Dermatol. 2000;143: 804-10.

7 Finasteride Male Pattern Hair Loss Study Group. Long-term (5-year) multinational experience with finasteride $1 \mathrm{mg}$ in the treatment of men with androgenetic alopecia. Eur J Dermatol. 2002;12:38-49. 
8 Kawashima M, Hayashi N, Igarashi A, Kitahara H, Maeguchi M, Mizuno A, et al. Finasteride in the treatment of Japanese men with male pattern hair loss. Eur J Dermatol. 2004;14:247-54.

9 Price VH, Menefee E, Sanchez M, Kaufman KD. Changes in hair weight in men with androgenetic alopecia after treatment with finasteride ( $1 \mathrm{mg}$ daily): three- and 4-year results. J Am Acad Dermatol. 2006;55:71-4.

10 Kaufman KD, Rotonda J, Shah AK, Meehan AG. Long-term treatment with finasteride 1 mg decreases the likelihood of developing further visible hair loss in men with androgenetic alopecia (male pattern hair loss). Eur J Dermatol. 2008;18:400-6.

11 Sato A, Takeda A. Evaluation of efficacy and safety of finasteride $1 \mathrm{mg}$ in 3177 Japanese men with androgenetic alopecia. J Dermatol. 2012;39:27-32.

12 Ricci G, Martinelli M, Luppi S, Lo Bello L, De Santis M, Skerk K, et al. Finasteride and fertility: case report and review of the literature. J Drugs Dermatol. 2012;11(12):1511-3.

13 Liu KE, Binsaleh S, Lo KC, Jarvi K. Propeciainduced spermatogenic failure: a report of two cases. Fertil Steril. 2008;90(3):849.

14 Altomare G, Capella GL. Depression circumstantially related to the administration of finasteride for androgenetic alopecia. J Dermatol. 2002;29(10):665-9.

15 Traish AM, Hassani J, Guay AT, Zitzmann M, Hansen ML. Adverse side effects of $5 a$-reductase inhibitors therapy: persistent diminished libido and erectile dysfunction and depression in a subset of patients. J Sex Med. 2011;8(3):872-84.

16 Irwig MS, Kolukula S. Persistent sexual side effects of finasteride for male pattern hair loss. J Sex Med. 2011;8(6):1747-53.
17 Irwig MS. Persistent sexual side effects of finasteride: could they be permanent? J Sex Med. 2012;9(11):2927-32.

18 Ganzer CA, Jacobs AR, Iqbal F. Persistent sexual, emotional, and cognitive impairment post-finasteride: a survey of men reporting symptoms. Am J Mens Health. 2015;9(3): 222-8.

19 Ali AK, Heran BS, Etminan M. Persistent sexual dysfunction and suicidal ideation in young men treated with low-dose finasteride: a pharmacovigilance study. Pharmacotherapy. 2015;35(7):687-95.

20 Irwig MS. Depressive symptoms and suicidal thoughts among former users of finasteride with persistent sexual side effects. J Clin Psychiatry. 2012;73(9):1220-3.

21 Overstreet JW, Fuh VL, Gould J, Howards SS, Lieber MM, Hellstrom W, et al. Chronic treatment with finasteride daily does not affect spermatogenesis or semen production in young men. J Urol. 1999 Oct;162(4):1295300.

22 Nguyen DD, Marchese M, Cone EB, Paciotti $\mathrm{M}$, Basaria S, Bhojani N, et al. Investigation of suicidality and psychological adverse events in patients treated with finasteride. JAMA Dermatol. 2021 Jan 1;157(1):35-42.

23 Fertig R, Shapiro J, Bergfeld W, Tosti A. Investigation of the plausibility of 5-alpha-reductase inhibitor syndrome. Skin Appendage Disord. 2017;2(3-4):120-9.

24 Grimalt R. Post-finasteride syndrome (CS23). Kyoto, Japan: 10th World Congress for Hair Research; 2017.

25 Rezende HD, Dias MFRG, Trüeb RM. A comment on the post-finasteride syndrome. Int $J$ Trichology. 2018 Nov-Dec;10(6):255-61.
26 Trüeb RM, Régnier A, Dutra Rezende H, Reis Gavazzoni Dias MF. Post-finasteride syndrome: an induced delusional disorder with the potential of a mass psychogenic illness? Skin Appendage Disord. 2019 Aug;5(5):3206.

27 Maffei C, Fossati A, Rinaldi F, Riva E. Personality disorders and psychopathologic symptoms in patients with androgenetic alopecia. Arch Dermatol. 1994;130(7):868-72.

28 LeFevre M. A role for finasteride in the prevention of prostate cancer? N Engl J Med 2013 Aug 15;369(7):670-1

29 Ramot Y, Czarnowicki T, Zlotogorski A. Finasteride induced gynecomastia: case report and review of the literature. Int J Trichology. 2009 Jan;1(1):27-9.

30 Basaria S, Jasuja R, Huang G, Wharton W, Pan $\mathrm{H}$, Pencina K, et al. Characteristics of men who report persistent sexual symptoms after finasteride use for hair loss. J Clin Endocrinol Metab. 2016;101(12):4669-80.

31 Bird ST, Brophy JM, Hartzema AG, Delaney JA, Etminan M. Male breast cancer and $5 a$-reductase inhibitors finasteride and dutasteride. J Urol. 2013 Nov;190(5):1811-4.

32 Kjærulff TM, Ersbøll AK, Green A, Emneus $\mathrm{M}$, Brasso K, Iversen $\mathrm{P}$, et al. Finasteride use and risk of male breast cancer: a case-control study using individual-level registry data from Denmark, Finland, and Sweden. Cancer Epidemiol Biomarkers Prev. 2019 May;28(5): 980-6.

33 Mondaini N, Gontero P, Giubilei G, Lombardi G, Cai T, Gavazzi A, et al. Finasteride $5 \mathrm{mg}$ and sexual side effects: how many of these are related to a nocebo phenomenon? J Sex Med. 2007;4:1708-12.

34 Groopman J. How doctors think. Boston/ New York: Houghton Mifflin Company; 2007. 